\title{
Responses of Acetylcholine Release and Regional Blood Flow in the Hippocampus during Walking in Aged Rats
}

\author{
Sae UCHIDA1, Atsuko SUZUKI2 ${ }^{2}$ Fusako KAGITANI'1, and Harumi HOTTA1 \\ 1Department of the Autonomic Nervous System, Tokyo Metropolitan Institute of Gerontology, Itabashi-ku, Tokyo, 173-0015 Japan; and \\ 2Laboratory of Physiology, Health Science University, Yamanashi, 401-0380 Japan
}

\begin{abstract}
Walking produced increases in both the extracellular acetylcholine level and regional blood flow in the hippocampus in aged rats 26-29 months old. The present results in aged rats were compared with our previous data in young adult rats (Naka-
\end{abstract}

jima et al., 2003), and it was found that both the acetylcholine and blood flow responses in the hippocampus were well maintained in aged rats.

Key words: aging, hippocampal blood flow, walking.

$\mathrm{O}$ ur laboratory demonstrated that upon the activation of cholinergic fibers that originate in the septal complex (i.e., the medial septal nucleus and the nucleus of the diagonal band) in the basal forebrain and project to the hippocampus, they release acetylcholine (ACh) in the hippocampus, resulting in an increase in regional cerebral blood flow in the hippocampus (Hpc CBF) via an activation of nicotinic ACh receptors (nAChRs) in anesthetized rats [1-4]. Further, we showed that such cholinergic Hpc CBF responses were activated during walking in conscious rats 3-4 months of age [5]. In that study, we found that walking at a moderate speed of $4 \mathrm{~cm} / \mathrm{s}$ increased $\mathrm{ACh}$ release in the hippocampus and produced an increase in $\mathrm{Hpc} \mathrm{CBF}$ accompanied by a small increase in the systemic mean arterial pressure (MAP). The increase in Hpc CBF during walking was attenuated by mecamylamine, a nAChR antagonist permeable to the blood brain barrier (BBB), but not by hexamethonium (C6), a nAChR antagonist impermeable to the BBB (a ganglionic blocker), though the walking-induced increase in MAP was abolished by either agent. These results suggest that the increase in $\mathrm{Hpc} \mathrm{CBF}$ during walking is independent of the pressor response and is attributable to an activation of the cholinergic vasodilator nerves projecting to the hippocampus [5].

Cholinergic neurons in the basal forebrain degenerate in patients with Alzheimer's disease [6] and in healthy elderly people [7]. However, Zhou et al. [8] found that the vasodilative response of the hippocampus upon stimulation of the septal complex was not significantly reduced in aged rats 28-29 months old. It would be interesting to know whether the intracranial cholinergic vasodilative system in the hippocampus is functioning during walking in aged rats. Thus the present study examined the effect of walking on extracellular ACh release and regional cerebral blood flow in the hippocampus of aged rats 26-29 months old by employing the same experimental procedures as those utilized in our previous study using young adult rats [5].

\section{Methods}

Ten healthy, aged, male Wistar rats (310-440 g, 26-29 months old) were used in the experiments. All animals were obtained from the animal farm of the Tokyo Metropolitan Institute of Gerontology. This study was approved by the Animal Committee of our institution. Among the 10 rats, 6 were used for the measurement of $\mathrm{Hpc} \mathrm{CBF}$, and the remaining 4 were used for the measurement of extracellular ACh level in the hippocampus. All experimental conditions, the technique of recording the MAP and Hpc CBF using laser Doppler flowmetry, and the technique of collecting and measuring extracellular $\mathrm{ACh}$ in the hippocampus using a microdialysis method and high-performance liquid chromatography with an electrochemical detector were the same as those described by Nakajima et al. [5]. The recovery rate of ACh by the microdialysis probe in vitro was $21-27 \%$ at room temperature. This range of recovery rate was the same as that in our previous study in young adult rats [5]. The rat was placed on a treadmill, and the treadmill was manually moved at a speed of approximately 2,4 , or $8 \mathrm{~cm} / \mathrm{s}$ for a 3 -min or 30 -s period, as described previously $[5,9]$. Hexamethonium (hexamethonium bromide, 10-20 mg/kg; Sigma, St. Louis, Missouri, USA), a ganglionic blocker, was administered intravenously through the caudal vein of the tail.

Received on Mar 6, 2006; accepted on Jun 6, 2006; released online on Jun 8, 2006; doi:10.2170/physiolsci.SC001706 Correspondence should be addressed to: Sae Uchida, Department of the Autonomic Nervous System, Tokyo Metropolitan Institute of Gerontology, 35-2 Sakae-cho, Itabashi-ku, Tokyo, 173-0015 Japan. Phone: +81-3-3964-3241 (Ext. 3086), Fax: +81-3-3579-4776, Email: suchida@tmig.or.jp 
The results are expressed as means \pm SEM. A statistical analysis was performed by an analysis of variance (ANOVA) followed by Dunnett's multiple comparison test, and a Student's $t$-test or two-way repeated ANOVA followed by Bonferroni post-test. Statistical significance was set at $p<0.05$.

\section{Results and discussion}

Response of $\mathrm{ACh}$ release to walking. The level of $\mathrm{ACh}$ in the hippocampus was measured every $3 \mathrm{~min}$ in 4 aged rats. The level of extracellular ACh under resting conditions before walking was $126 \pm 29 \mathrm{fmol} / 3 \mathrm{~min}$ in the aged rats. During the resting condition of $9 \mathrm{~min}$, the level of extracellular ACh was stable in each animal. Comparing the present data from aged male rats with our previous data from young adult male rats [5], in which extracellular ACh release in the hippocampus under the conscious and resting condition was $109 \pm 22 \mathrm{fmol} / 3 \mathrm{~min}$, there was no significant difference in the level of ACh release between the two groups.

In the aged rats, walking at $4 \mathrm{~cm} / \mathrm{s}$ for 3 min significantly increased ACh release, reaching $238 \pm 23 \%$ of the prewalking control value, and then the extracellular ACh level gradually returned to the basal level (Fig. 1). In a comparison of the present data from aged rats with our previous data from young adult rats [5], there were no significant differences in the magnitude or time course of a walking-induced increase in the ACh release.

This result suggests that the walking-induced release of $\mathrm{ACh}$ in the hippocampus from cholinergic terminals that probably originate in the septal complex is well maintained in the healthy aged rats when tested with moderatespeed walking. There have been conflicting results on age-related changes in the basal and evoked release of $\mathrm{ACh}$ in the hippocampus in conscious rats; Scali et al. [10] reported that scopolamine caused a larger increase in hippocampal ACh release in young rats than in old ones, but Fischer et al. [11] reported that hippocampal ACh release was unchanged in old rats compared with young ones. There are also conflicting results concerning the age-related deaths of cholinergic neurons in the septal complex in rats; one study reported that the number of neurons was decreased in the aged rats [12], and another found that the number of neurons was not changed [13]. Fischer et al. [11] reported that the extracellular ACh release in the hippocampus in response to $\mathrm{K}^{+}$-induced depolarization and behavioral activation is maintained in the aged rats, but the cholinergic neurons in the septal complex showed a slight reduction in both number and size. Our results on ACh release may suggest that at least the healthy aged Wistar rats used in the present experiments do not have a marked degeneration of septohippocampal cholinergic neurons, or their septohippocampal cholinergic system compensates functionally for ongoing degeneration or atrophic processes.

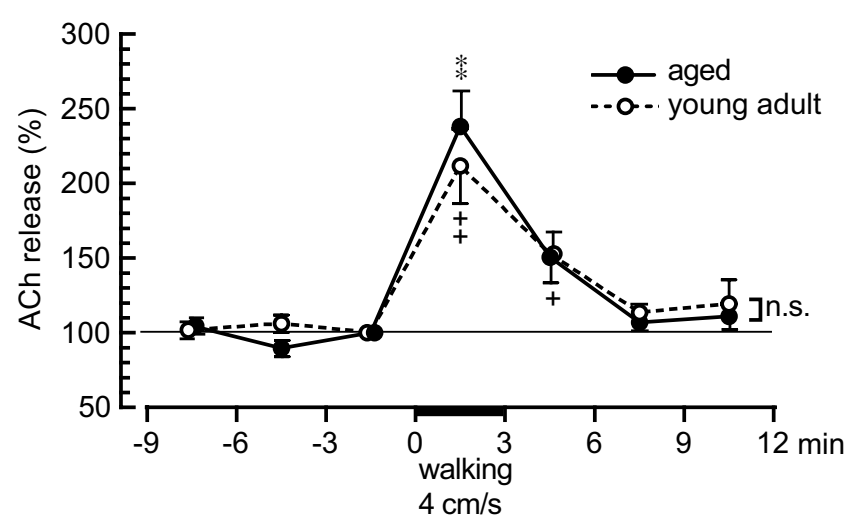

Fig. 1. Response of ACh release in the hippocampus to walking at $4 \mathrm{~cm} / \mathrm{s}$ for $3 \mathrm{~min}$ in aged (26-29 months old) rats. The level of extracellular $\mathrm{ACh}$, which was measured every $3 \mathrm{~min}$, is expressed as a percentage of the prewalking control values (from -3 to $0 \mathrm{~min}$ ) on the ordinate. The onset of walking was set at time zero on the abscissa. The underbar indicates the 3-min walking period. Each closed circle and vertical bar show the mean \pm SEM (4 aged animals). For each rat, 2 trials were averaged. The responses obtained from 4 young adult rats of 3-4 months of age in our previous study [5] are shown by open circles (mean) and vertical bars (SEM) for comparison. The symbols $*$ and $* *$ (for aged rats) and + and ++ (for young adult rats) represent statistically significant differences from the prewalking value (from -3 to $0 \mathrm{~min}$ ) at $p<0.05$ and $p$ $<0.01$, respectively, as determined by one-way repeated ANOVA, followed by Dunnett's multiple comparisons test. n.s.: There is no significant difference in the response of $\mathrm{ACh}$ release to walking between aged rats and young adult rats (two-way repeated ANOVA).

Response of hippocampal cerebral blood flow. The present method using laser Doppler flowmetry to measure Hpc CBF does not provide us with an absolute measurement of Hpc CBF, because the absolute level of flow depends on the contact conditions of the probe within the hippocampus in each rat. It has been reported that Hpc CBF does not differ significantly between conscious rats of 3-34 months old when measured using the $\left[{ }^{14} \mathrm{C}\right]$-iodoantipyrine method [14]. In the present study, the walking-induced response of Hpc CBF using laser Doppler flowmetry was expressed as a percentage of the resting value.

As shown in the sample recordings before, during, and after walking at the 3 different speeds of $2 \mathrm{~cm} / \mathrm{s}, 4 \mathrm{~cm} / \mathrm{s}$, and $8 \mathrm{~cm} / \mathrm{s}$ for a 30 -s period in an aged rat (Fig. 2, A-C), Hpc CBF (above recordings) and MAP (below recordings) started to increase within $1-2 \mathrm{~s}$ after the start of walking, and the increase nearly reached the maximum within approximately $10 \mathrm{~s}$ after the start. The increased Hpc CBF during walking lasted for 5-20 s after the end of walking, depending on the individual case, and the Hpc CBF level then gradually recovered to the control level. Walking was repeated after intrervals of 5-10 min, and the responses of $\mathrm{Hpc} \mathrm{CBF}$ and MAP were reproducible in each animal. Hpc $\mathrm{CBF}$ increased during walking at $2 \mathrm{~cm} / \mathrm{s}$ 
Fig. 2. Responses of $\mathrm{Hpc}$ $\mathrm{CBF}$ and MAP during walking at the three different speeds of $2 \mathrm{~cm} / \mathrm{s}$ (A, D), 4 $\mathrm{cm} / \mathrm{s}(\mathbf{B}, \mathbf{E})$, and $8 \mathrm{~cm} / \mathrm{s}(\mathbf{C}$, F) in aged (26-29 months old) rats. (A-C) Sample recordings in one rat. (D-F) Summary of the responses of $\mathrm{Hpc}$ CBF and MAP before, during, and after walking ( $n=$ 4). The mean values of $\mathrm{Hpc}$ CBF and MAP during a 30 -s period were plotted every 30 $\mathrm{s}$ as the percentage of the corresponding prewalking control values, from -30 to 0 $\mathrm{s}$. The onset of walking was set at time zero on the abscissa. Underbars indicate the 30-s walking period. Each closed circle and vertical bar show the mean \pm SEM (4 aged animals). For each rat, 2 trials were aver-
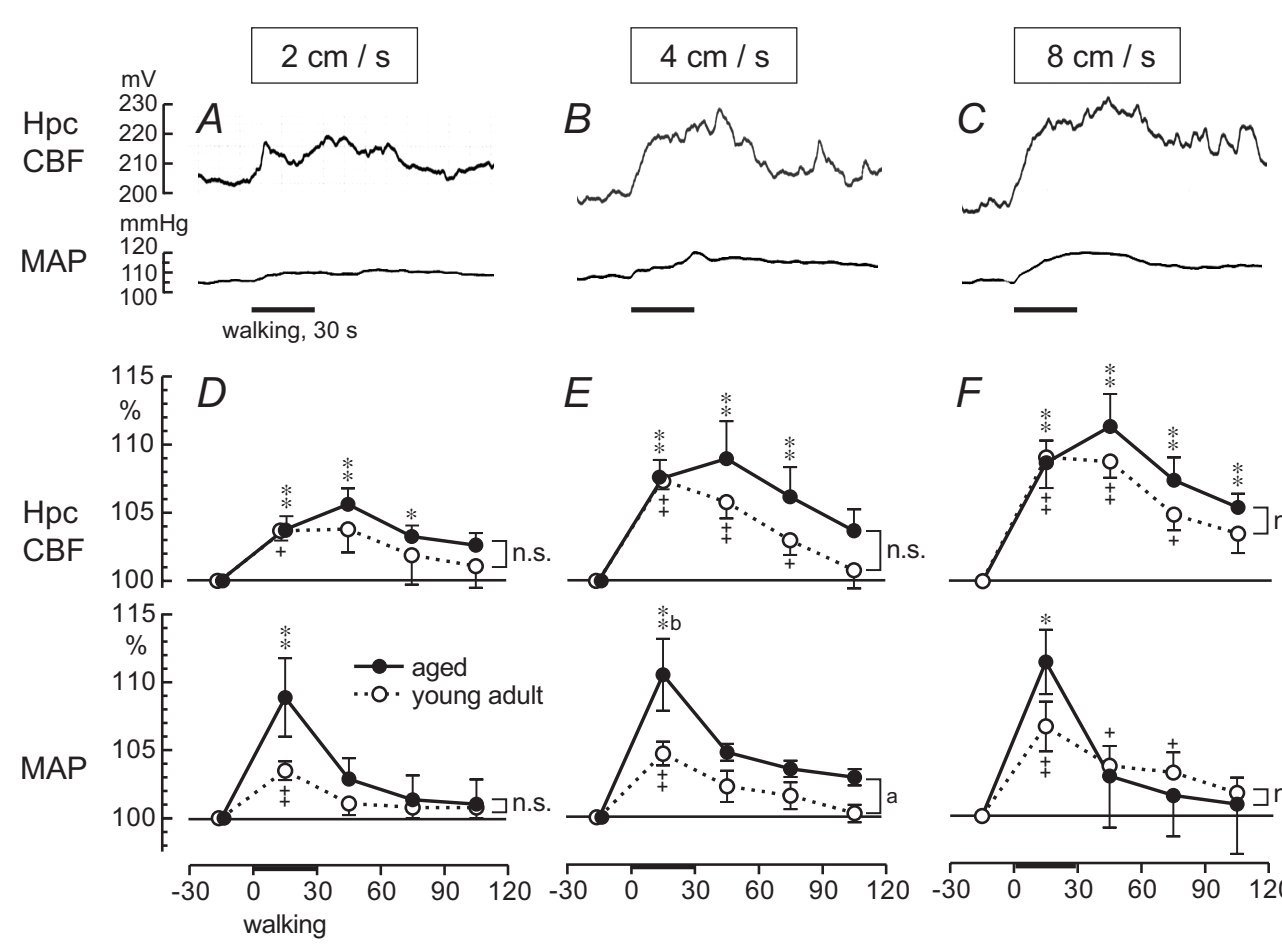

aged. The responses ob-

tained from 4 young adult rats 3-4 months old in our previous study [5] are shown by open circles (mean) and vertical bars (SEM) for comparison. The symbols $*$ and $* *$ (for aged rats) and + and ++ (for young adult rats) represent statistically significant differences from the prewalking control values at $p<0.05$ and $p<0.01$, respectively, as determined by one-way repeated ANOVA followed by Dunnett's multiple comparisons test. ap $<0.05$, significant difference between the aged rats and young adult rats, tested by two-way repeated ANOVA. ${ }^{\mathrm{b}} p<0.01$, significantly different from the respective values of young adult rats, tested by a Bonferroni post-test.

by $3.8 \pm 1.0 \%$ (Fig. 2D) and at $4 \mathrm{~cm} / \mathrm{s}$ by $7.6 \pm 1.3 \%$ (Fig. $2 \mathrm{E}$ ) in the aged rats. Hpc CBF was increased for another $60 \mathrm{~s}$ after the end of walking. Hpc CBF increased during walking at $8 \mathrm{~cm} / \mathrm{s}$ by $8.7 \pm 1.9 \%$ (Fig. $2 \mathrm{~F}$ ) and remained elevated for another $90 \mathrm{~s}$ after the end of walking. However, MAP increased only during walking, by $8.9 \pm 2.9 \%$, $10.5 \pm 2.6 \%$, and $11.3 \pm 2.4 \%$ at 2,4 , and $8 \mathrm{~cm} / \mathrm{s}$, respectively. The response of $\mathrm{Hpc} \mathrm{CBF}$ during walking at 2, 4, and $8 \mathrm{~cm} / \mathrm{s}$ lasted longer than the response of MAP. The magnitude of the increase in Hpc CBF during walking at 2,4 , and $8 \mathrm{~cm} / \mathrm{s}$ was dependent on the walking speed. Comparing the present data in aged rats with our previous data in young adult rats [5] concerning the increased response of $\mathrm{Hpc} \mathrm{CBF}$ during walking, there were no significant differences in the magnitude or time course at any walking speed. However, the magnitude of the increased response of MAP during walking tended to be larger in aged rats than in young adult rats at any walking speed, and it was significantly larger in aged rats than in young adult rats at a walking speed of $4 \mathrm{~cm} / \mathrm{s}$. In humans, it has also been reported that older subjects have a higher blood pressure response to exercise than their younger counterparts [15]. The variations of the responses of $\mathrm{Hpc}$ CBF and MAP were larger in aged rats than in young adult rats. It is well known that individual variability in physiologi- cal responses increases with age [16].

When walking duration was increased to $3 \mathrm{~min}$, the magnitude of increase in $\mathrm{Hpc}$ CBF during walking at a speed of $4 \mathrm{~cm} / \mathrm{s}$ became $12.7 \pm 2.1 \%$ in aged rats $(n=5)$ and $15.3 \pm 1.9 \%$ in young adult rats $(n=4$, unpublished data). During $3 \mathrm{~min}$ of walking at $4 \mathrm{~cm} / \mathrm{s}$, MAP increased $9.6 \pm 3.1 \%$ in aged rats $(n=5)$ and $6.8 \pm 1.5 \%$ in young adult rats $(n=4$, unpublished data). There were no significant differences between the two groups in both parameters.

To eliminate the pressor response during walking, the responses of $\mathrm{Hpc} \mathrm{CBF}$ and MAP to walking for $30 \mathrm{~s}$ at a speed of $4 \mathrm{~cm} / \mathrm{s}$ were examined before and after i.v. injection of C6, 10-20 mg/kg in 4 aged rats. The basal level of MAP before walking significantly decreased after C6 administration to $49 \pm 6 \mathrm{mmHg}$, from $94 \pm 7 \mathrm{mmHg}$. The significant increase in MAP during walking shown before C6 administration was abolished afterward (Fig. 3). The basal level of Hpc CBF before walking significantly decreased after C6 administration to $191 \pm 20 \mathrm{mV}$, from 240 $\pm 18 \mathrm{mV}$. The increase in Hpc CBF during walking and for $60 \mathrm{~s}$ after the end of walking was not significantly changed after $\mathrm{C} 6$ administration (Fig. 3). Thus Hpc CBF response in aged rats is not completely dependent on an increase in MAP, though the magnitude of the pressor re- 

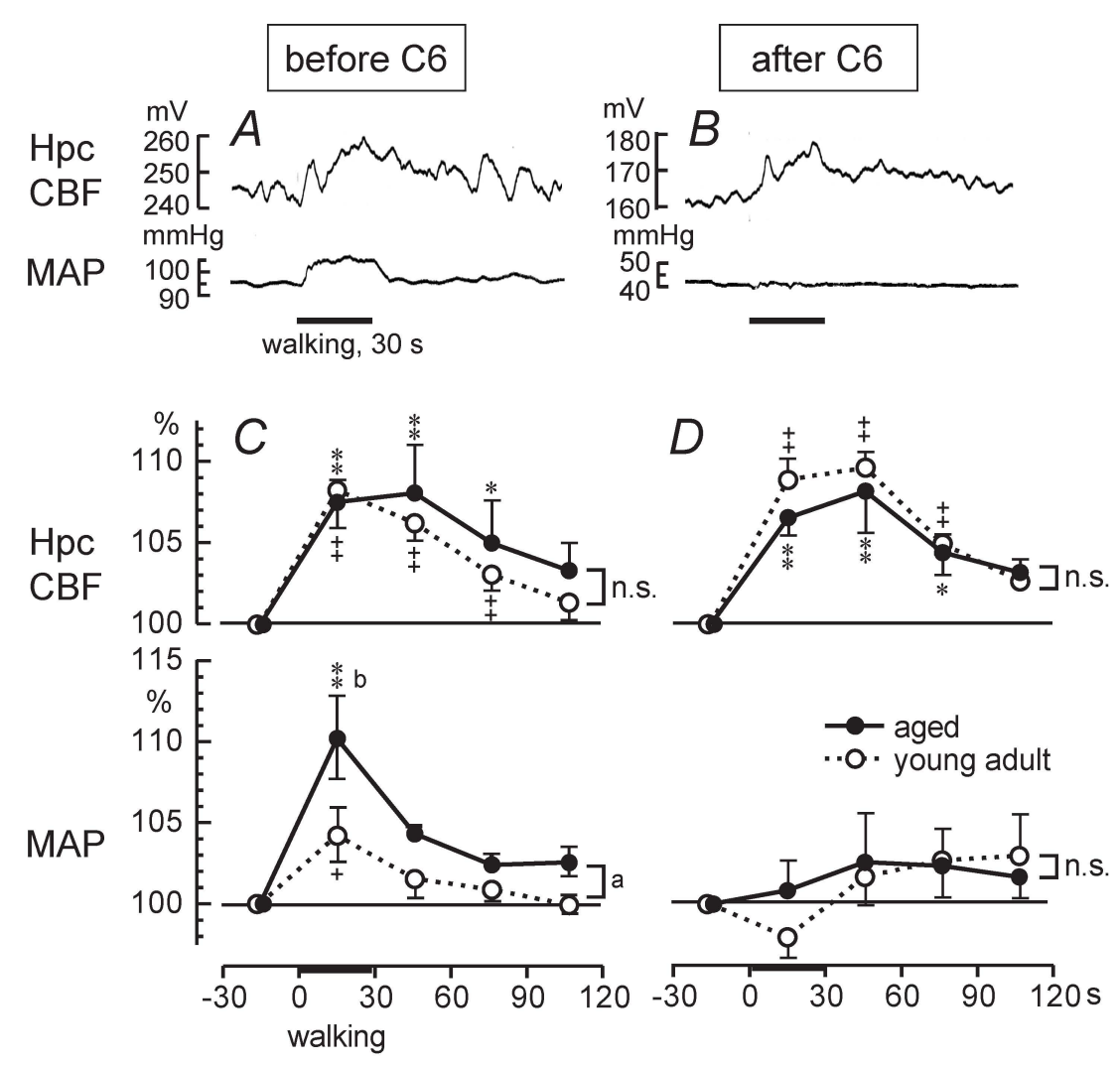

sponse during walking in aged rats is larger than in young adult rats. At least some parts of the increase in Hpc CBF were independent of the increase in MAP not only in young adult rats, but also in aged rats. The magnitude and time course of an increased response of $\mathrm{Hpc}$ CBF to walking after $\mathrm{C} 6$ administration in aged rats were similar to that in young adult rats (Fig. 3). Therefore the present results suggest that the portion of the vasodilative response of hippocampal blood vessels during walking that is independent of the pressor response is well maintained in aged rats when tested with moderate speed walking.

Our previous study in young adult rats suggested that the portion of the increase in Hpc CBF during walking that is independent of the change in MAP may be due to an activation of intracranial cholinergic vasodilator nerves projecting to the hippocampus [5]. In aged rats of 28-29 months, the cholinergic vasodilator response in the hippocampus to stimulation of the septal complex was well maintained [8]. Our present finding of an increase in Hpc CBF during walking that was independent of the change in MAP may suggest that the intracranial cholinergic vasodilative system in the hippocampus is functioning during walking in aged rats 26-29 months old.

The response of ACh release during walking was well maintained in healthy aged rats. The well-maintained vasodilative response of the hippocampal blood vessels during walking at a moderate speed of $4 \mathrm{~cm} / \mathrm{s}$ is probably achieved through an undiminished activation of the cholinergic receptors related to this vasodilative response [5].
Fig. 3. Effect of intravenous administration of $\mathrm{C} 6$ on the walking-induced responses of Hpc CBF and MAP in aged rats. (A-B) Sample recordings before $(A)$ and $29 \mathrm{~min}$ after (B) an administration of C6 $(10 \mathrm{mg} / \mathrm{kg}$, i.v.). (C-D) Summary of the responses to walking before (C) and 25-30 min after (D) an administration of C6 (10-20 mg/kg, i.v.; $n$ $=4$ ). For each rat, 2 trials were averaged. Other details are the same as in Fig. 2.
It was reported that spatial learning using the hippocampus is facilitated by running in young adult rats and in aged rats $[17,18]$. We assume that the increase in Hpc CBF during walking is involved in such facilitation of spatial learning using hippocampal activation. Furthermore, walking-induced increases in Hpc CBF provide increased amounts of oxygen and glucose to the hippocampus, and these supplemental nourishments appear to be advantageous for the neurons of the hippocampus, such as for protection against delayed neuronal death resulting from insufficient blood supply, as reported in adult rats [19].

It was reported that in elderly people, exercise is useful to maintain their cognitive functions [20,21]. Rogers et al. [22] reported that in elderly people, physical activity including walking prevents the age-related decline of cerebral perfusion and of cognitive functions. Future studies to confirm that the intracranial cholinergic vasodilative system is activated in elderly persons during walking are warranted.

We are grateful to Professor Akio Sato of the University of Human Arts and Sciences for his encouragement to complete this study.

\section{REFERENCES}

1. Cao $\mathrm{W}-\mathrm{H}$, Inanami $\mathrm{O}$, Sato $A$, Sato $Y$. Stimulation of the septal complex increases local cerebral blood flow in the hippocampus in anesthetized rats. Neurosci Lett. 1989;107:135-40.

2. Adachi T, Inanami O, Ohno K, Sato A. Responses of regional cerebral blood flow following focal electrical stimulation of the nucleus basalis of Meynert and the medial septum using the $\left[{ }^{14} \mathrm{C}\right]$ iodoantipyrine method in rats. Neurosci Lett. 
1990;112:263-8.

3. Sato A, Sato Y. Regulation of regional cerebral blood flow by cholinergic fibers originating in the basal forebrain. Neurosci Res. 1992;14:242-74.

4. Sato $A$, Sato Y. Cholinergic neural regulation of regional cerebral blood flow. Alzheimer Dis Assoc Disord. 1995;9:28-38.

5. Nakajima K, Uchida S, Suzuki A, Hotta H, Aikawa Y. The effect of walking on regional blood flow and acetylcholine in the hippocampus in conscious rats. Auton Neurosci. 2003;103:83-92.

6. Whitehouse PJ, Price DL, Struble RG, Clark AW, Coyle JT, DeLong MR. Alzheimer's disease and senile dementia: loss of neurons in the basal forebrain. Science. 1982;215:1237-9.

7. McGeer PL, McGeer EG, Suzuki J, Dolman CE, Nagai T. Aging, Alzheimer's disease, and the cholinergic system of the basal forebrain. Neurology. 1984;34:741-5

8. Zhou W, Cao W-H, Sato A. Vasodilator responses in the hippocampus in response to stimulation of the septal complex are well maintained in aged rats (in Japanese). Auton Nerv Syst. 1990;27:552-7.

9. Kimura A, Okada K, Sato A, Suzuki H. Regional cerebral blood flow in the frontal, parietal and occipital cortices increases independently of systemic arterial pressure during slow walking in conscious rats. Neurosci Res. 1994;20:309-15.

10. Scali C, Vannucchi MG, Pepeu G, Casamenti F. Peripherally injected scopolamine differentially modulates acetylcholine release in vivo in the young and aged rats. Neurosci Lett. 1995;197:171-4.

11. Fischer W, Nilsson OG, Björklund A. In vivo acetylcholine release as measured by microdialysis is unaltered in the hippocampus of cognitively impaired aged rats with degenerative changes in the basal forebrain. Brain Res. 1991;556:4452.
12. de Lacalle S, Cooper JD, Svendsen CN, Dunnett SB, Sofroniew MV. Reduced retrograde labelling with fluorescent tracer accompanies neuronal atrophy of basal forebrain cholinergic neurons in aged rats. Neuroscience. 1996;75:19-27.

13. Gibbs RB. Impairment of basal forebrain cholinergic neurons associated with aging and long-term loss of ovarian function. Exp Neurol. 1998;151:289-302.

14. Ohata M, Sundaram U, Fredericks WR, London ED, Rapoport SI. Regional cerebral blood flow during development and ageing of the rat brain. Brain. 1981;104:319-32.

15. Julius $S$, Amery A, Whitlock LS, Conway J. Influence of age on the hemodynamic response to exercise. Circulation. 1967;36:222-30.

16. Timiras PS. Developmental Physiology and Aging. New York: The Macmillan Company; 1972.

17. Fordyce DE,Farrar RP. Physical activity effects on hippocampal and parietal cortical cholinergic function and spatial learning in F344 rats. Behav Brain Res. 1991;43:115-23.

18. Albeck DS, Sano K, Prewitt GE, Dalton L. Mild forced treadmill exercise enhances spatial learning in the aged rat. Behav Brain Res. 2006;168:345-8.

19. Kagitani F, Uchida S, Hotta H, Sato A. Effects of nicotine on blood flow and delayed neuronal death following intermittent transient ischemia in rat hippocampus. Jpn J Physiol. 2000;50:585-95.

20. Yaffe K, Barnes D, Nevitt M, Lui L-Y, Covinsky K. A prospective study of physical activity and cognitive decline in elderly women: women who walk. Arch Intern Med. 2001;161:1703-8

21. Kramer AF, Colcombe SJ, McAuley E, Scalf PE, Erickson KI. Fitness, aging and neurocognitive function. Neurobiol Aging. 2005;26S:S124-7.

22. Rogers RL, Meyer JS, Mortel KF. After reaching retirement age physical activity sustains cerebral perfusion and cognition. J Am Geriatr Soc. 1990;38:123-8. 